\title{
Surface Control of the Frequency of Stratospheric Sudden Warming Events
}

\author{
ZHENG WU AND THOMAS REICHLER \\ Department of Atmospheric Sciences, University of Utah, Salt Lake City, Utah
}

(Manuscript received 22 November 2018, in final form 15 March 2019)

\begin{abstract}
The frequency of stratospheric sudden warming events (SSWs) is an important characteristic of the coupled stratosphere-troposphere system. However, many modern climate models are unable to reproduce the observed SSW frequency. A previous study suggested that one of the reasons could be the momentum damping at the surface. The goal of the present study is to understand what determines the climatological SSW frequency and how the surface damping comes into play. To this end, we conduct a parameter sweep with an idealized model, using a wide range of values for the surface damping. It is found that the SSW frequency is a strong and nonlinear function of the surface damping. Various tropospheric and stratospheric factors are identified to link the surface damping to the SSW frequency. The factors include the magnitude of the surface winds, the meridional and vertical wind shear, the synoptic eddy activity in the troposphere, the transient wave activity flux at the lower stratosphere, and the strength of the stratospheric polar vortex. Mathematicalstatistical modeling, informed by the parameter sweep, is used to quantify how the different factors relate to each other. This successfully reproduces the complex variations of the SSW frequency with the surface damping seen in the parameter sweep. The results may help in explaining some of the difficulties that climate models have in simulating the observed SSW frequency.
\end{abstract}

\section{Introduction}

Stratospheric sudden warming events (SSWs) are the most dramatic large-scale circulation events to occur in the winter Arctic stratosphere and are classical examples of strong wave-mean flow interaction (McIntyre 1982). There is some debate on the relative influences of tropospheric and stratospheric conditions for the development of individual SSWs (e.g., Sun et al. 2012; Jucker 2016; de la Cámara 2017), but it is generally accepted that strong pulses of upward propagating planetary wave activity (Matsuno 1971; Charlton et al. 2007; Coy et al. 2009; Limpasuvan et al. 2004; Polvani and Waugh 2004) combined with a relatively weak stratospheric polar vortex (Horan and Reichler 2017; Limpasuvan et al. 2004; Scott and Polvani 2006) provide favorable conditions for the occurrence of SSWs.

SSW events are rare and happen on average in only two out of every three years. SSW events are also extreme and are important for the mean and variability of stratospheric climate and for the dynamical coupling between the stratosphere and troposphere (Baldwin and Dunkerton 2001). How often SSW events occur in a

Corresponding author: Zheng Wu, zheng.winnie.wu@utah.edu climatological sense is therefore an essential characteristic of the extratropical stratosphere-troposphere system. Given this importance it is surprising that the factors that determine the climatological SSW frequency are not very well understood. Perhaps even more surprising is that the SSW frequency simulated by climate models varies widely and that the reasons behind such variations are also largely unclear. For example, Charlton et al. (2007) found variations between $10 \%$ and $79 \%$ in six climate models, SPARC (2010) reported variations between $20 \%$ and $100 \%$ in 14 chemistry-climate models, and Charlton-Perez et al. (2013) found frequencies between $0 \%$ and $80 \%$ in a large number of CMIP5 models.

Nevertheless, previous studies provide some clues about what influences the SSW frequency in models. For example, Charlton-Perez et al. (2013) demonstrated that a sufficient vertical resolution and a reasonable stratospheric circulation are important requirements: models with a relatively low model lid (below $1 \mathrm{hPa}$ ) tend to severely underestimate the SSW frequency. Along similar lines, Lee and Black (2015) showed that the variability of both the stratospheric planetary wave activity and the polar vortex strength is underestimated in the CMIP5 low-top models. Shaw and Perlwitz (2010) 
noted that the low-top version of the Canadian Middle Atmosphere Model underestimates extreme heat flux events and SSWs, and that this is due to excessive damping near the model lid. But vertical resolution alone is not sufficient, as the SSW frequencies in stratosphere-resolving models also show large spread (SPARC 2010; Charlton et al. 2007). Charlton et al. (2007) found that in models with too few warmings the mean and variability of the meridional heat flux in the lower stratosphere are too low, suggesting that not enough tropospheric Rossby wave activity could be a reason. They also suggested that the climatological strength of the polar vortex is important, as models with a too strong vortex tended to have too few SSWs. Charlton et al. (2007) also hinted that the simulated structure of the climatological stratospheric winds is important, because the winds determine the refractive properties for the resolved waves.

Another class of dynamical models, so-called idealized dry-dynamical core models (Held and Suarez 1994), have also been popular tools for the investigation of SSWs. Simulations with such models found that the height and structure of the surface topography is important, with larger topographic amplitudes and a wavenumber- 2 structure leading in general to a weaker and more variable stratospheric vortex and hence more SSWs than a small-amplitude wavenumber-1 topography (Taguchi et al. 2001; Gerber and Polvani 2009; Jucker et al. 2014; Sheshadri et al. 2015; Martineau et al. 2018). On the other hand, Lindgren et al. (2018) demonstrated that with appropriate choices of tropospheric heating perturbations, this kind of simple model can also produce a reasonable number of SSWs without the use of topographic forcing. As with complex models, the climatological strength of the polar vortex also influences the SSW frequency in idealized models. For example, Gerber and Polvani (2009) found that a very weak vortex reduces the variability of the vortex and the number of SSWs. On the other hand, if the vortex is very strong, it is increasingly difficult for the zonal-mean zonal wind to reverse, decreasing the number of SSWs despite an increase in vortex variability (Jucker et al. 2014; Gerber and Polvani 2009). The inclusion of a seasonal cycle (Sheshadri et al. 2015) and the spatial structure of the damping time scale (Jucker et al. 2014; Hitchcock and Shepherd 2013) also influence SSWs. Martineau et al. (2018) pointed out the important role of the lower-stratospheric basic state in controlling the strength and variability of the stratospheric polar vortex. In their study, a colder lower stratosphere favored a stronger vortex and an enhanced upward propagation of wave activity with a broader distribution, leading to larger stratospheric variability and more SSWs. Similar to Gerber and Polvani (2009), it was argued that the lower stratosphere is important because the winds and potential vorticity gradients are weak in this region, potentially limiting the strength of upward propagating bursts of wave activity into the vortex region.

The magnitude of the momentum damping at the surface and the lower troposphere (hereafter simply called surface drag) is another interesting factor for the SSW frequency. For example, Richter et al. (2010) found that the inclusion of additional drag in their WACCM 3.5 model led to dramatic improvements (from $10 \%$ to $40 \%$ ) in the SSW frequency. The extra drag led to a decrease in surface winds by up to $\sim 2 \mathrm{~m} \mathrm{~s}^{-1}$ and reduction in the generation of orographic gravity waves, strengthening the westerlies in the lower stratosphere. The wind change modified the refraction of upward propagating planetary waves and increased the amount of wave activity directed into the polar vortex. In idealized models, where gravity wave drag is only crudely parameterized in a sponge layer at the model top, other influences from the surface drag are thinkable. For example, changes in drag modify the strength and latitudinal position (Chen et al. 2007) of the tropospheric westerlies and possibly the interaction of the westerlies with the topography. Moreover, zonal asymmetries in drag, if included in a model to simulate the different surface characteristics of land and ocean, may have similar effects on the westerlies. The shifts in the winds associated with the drag changes may impact the amplitude and position of the stationary waves and the tropospheric wave activity, the wave driving of the stratosphere, and therefore the frequency of SSWs.

The goal of the present paper is to further explore the above ideas about the possible role of the surface drag for the frequency of SSWs in an idealized model setting. In doing so, we hope to not only provide new insight into the regulators for the climatological SSW frequency but also contribute to a deeper understanding for the dynamics of the coupled stratosphere-troposphere system in general. The present study is motivated by a recent paper of us (Wu and Reichler 2018, hereafter WR18), in which we employed an idealized model with realistic topography and zonally asymmetric equilibrium temperatures to probe the sensitivity of the stratospheric circulation to variations in surface drag. We found an interesting sensitivity of the simulated frequency of SSWs to the magnitude of the prescribed momentum damping at the model's lower boundary, which we want to better understand. Consequently, we use in the present study the same model to conduct a parameter sweep in which we vary the magnitude of the surface drag over a wide range of values. We use the experiments to explore the relationship between drag and SSW frequency, and as we 
will show in this paper, we find that the frequency is related to the strength of the surface drag in very nonlinear ways. We use the results to construct a simple dynamical framework that explains this relationship.

The paper is organized as follows. In section 2, we describe the model setup, the numerical experiments, and our diagnostic methods. In section 3, we explore the sensitivity of the stratospheric and tropospheric circulations to the surface momentum damping, with an emphasis on understanding the impacts on the SSW frequency. We formulate a simple mathematical-statistical model that explains and quantifies the nonlinear relationship between drag and SSW frequency. Based on surface drag alone, the model describes the variations of several dynamical quantities that are key to explain the SSW frequency. Finally, section 4 provides our conclusions and a discussion.

\section{Numerical experiments and methods}

\section{a. Model setup and experimental design}

We use the spectral dynamical core of the Geophysical Fluid Dynamics Laboratory (GFDL) (Held and Suarez 1994) with a horizontal resolution of T42 and 40 vertical $\sigma$ levels between the surface and $0.01 \mathrm{hPa}$. The model nudges temperatures toward a prescribed equilibrium temperature $T_{\text {eq }}$ by Newtonian relaxation to mimic diabatic heating in the form of $(\partial T / \partial t)=-(T-$ $\left.T_{\text {eq }}\right) / \tau$, where $T$ is temperature and $\tau$ is a prescribed relaxation time scale. The $T_{\text {eq }}$ fields are determined individually for each month of the year and then linearly interpolated to daily values. As explained below, each of our experiments uses a different $T_{\text {eq }}$ field. The relaxation time scale $\tau$ is kept the same for all experiments and is taken from the analytical expression of Jucker et al. (2014), given in their appendix. The expression mimics observationally derived values. Note that $\tau$ is zonally symmetric and latitude and pressure dependent, decaying in height over the high latitudes from 40 days in the troposphere to 5 days in the upper stratosphere. The model uses Rayleigh friction to simulate momentum damping in the planetary boundary layer. This can be written as $\partial v / \partial t=\cdots-k_{v}(\sigma) v$, where $v$ is the wind, $\sigma$ is the model's vertical sigma level $p / p_{s}$, and $k_{v}$ is the prescribed momentum damping rate; $k_{v}$ decreases linearly with height, from a specified value at the surface $(\sigma=1$, denoted as the surface drag, or $D_{S}$ ) to a value of zero at $\sigma=0.7$. Linear drag is also applied in a sponge layer at the model top (above $0.15 \mathrm{hPa}$ ) to approximate the drag from the breaking of unresolved gravity waves.

Most previous studies used a highly idealized (or no) topography and zonally symmetric $T_{\text {eq }}$ to run this type of model. In contrast, in our model we employ realistic
Earth-like topography and introduce zonal asymmetries into $T_{\mathrm{eq}}$ following a procedure proposed by Chang (2006). The procedure minimizes the climatological temperature difference between the MERRA-2 reanalysis (Bosilovich et al. 2016) and the model by iteratively adjusting $T_{\text {eq }}$. Starting from some $T_{\text {eq }}$ (Jucker et al. 2014), we use the climatological temperature difference between the model and the reanalysis to correct $T_{\text {eq }}$ and start a new iteration. This way, the model's four-dimensional (longitude $\times$ latitude $\times$ height $\times$ month) temperature climatology converges toward the reanalysis. As discussed in WR18, an important outcome is that the model's effective diabatic heating rate $Q=\left(T_{\mathrm{eq}}-\bar{T}\right) / \tau$ resembles that of the reanalysis and that the spectrum of the planetary waves generated by the model is more realistic compared to earlier approaches. More details about the iterative procedure are given in WR18.

We use the WR18 model to perform a parameter sweep of surface drag $D_{S}$. The sweep consists of $20 \mathrm{ex}-$ periments differing only in the value of $D_{S}$ and its associated $T_{\text {eq }}$, with $\tau$ kept constant. As shown in Fig. 1a, $D_{S}$ ranges between 0 and 5.6 day $^{-1}$. We note that some of the $D_{S}$ values are unrealistically small or large, but we intentionally chose this wide range to be better able to test our ideas. We also note that Held and Suarez (1994) originally proposed $D_{S}=1.0 \mathrm{day}^{-1}$.

Eleven of the 20 experiments are taken from our WR18 study. In addition, we conduct nine extra experiments; $T_{\text {eq }}$ for each experiment is individually and iteratively determined so that all experiments produce a nearly identical reanalysis-like temperature climatology. In determining $T_{\text {eq }}$, we conduct 31 iterations for most experiments. Each iteration is run for 60 years, except for the last one, which is run for 501 years to facilitate an improved statistical analysis. After the final iteration, the root-mean-square (RMS) error between each experiment's three-dimensional temperature climatology $\bar{T}$ and the MERRA-2 climatology $\overline{T_{C}}$ is $\sim 1.2 \mathrm{~K}$. Further reductions are difficult to achieve because of the smallness of the surface drag for experiments with $D_{S}<0.6$ day $^{-1}$, resulting in quite unrealistic winds and larger temperature errors.

\section{b. Diagnostics}

Our analysis is carried out for January-March (JFM) means because this is when most SSWs happen (Horan and Reichler 2017). We calculate Northern Hemisphere $(\mathrm{NH})$ area means by taking longitudinal averages and latitude-weighted averages from $20^{\circ}$ to $90^{\circ} \mathrm{N}$, unless specified otherwise. In defining SSWs, we apply the "WMO" criterion (McInturff 1978; Charlton and Polvani 2007), which is based on a reversal of the 

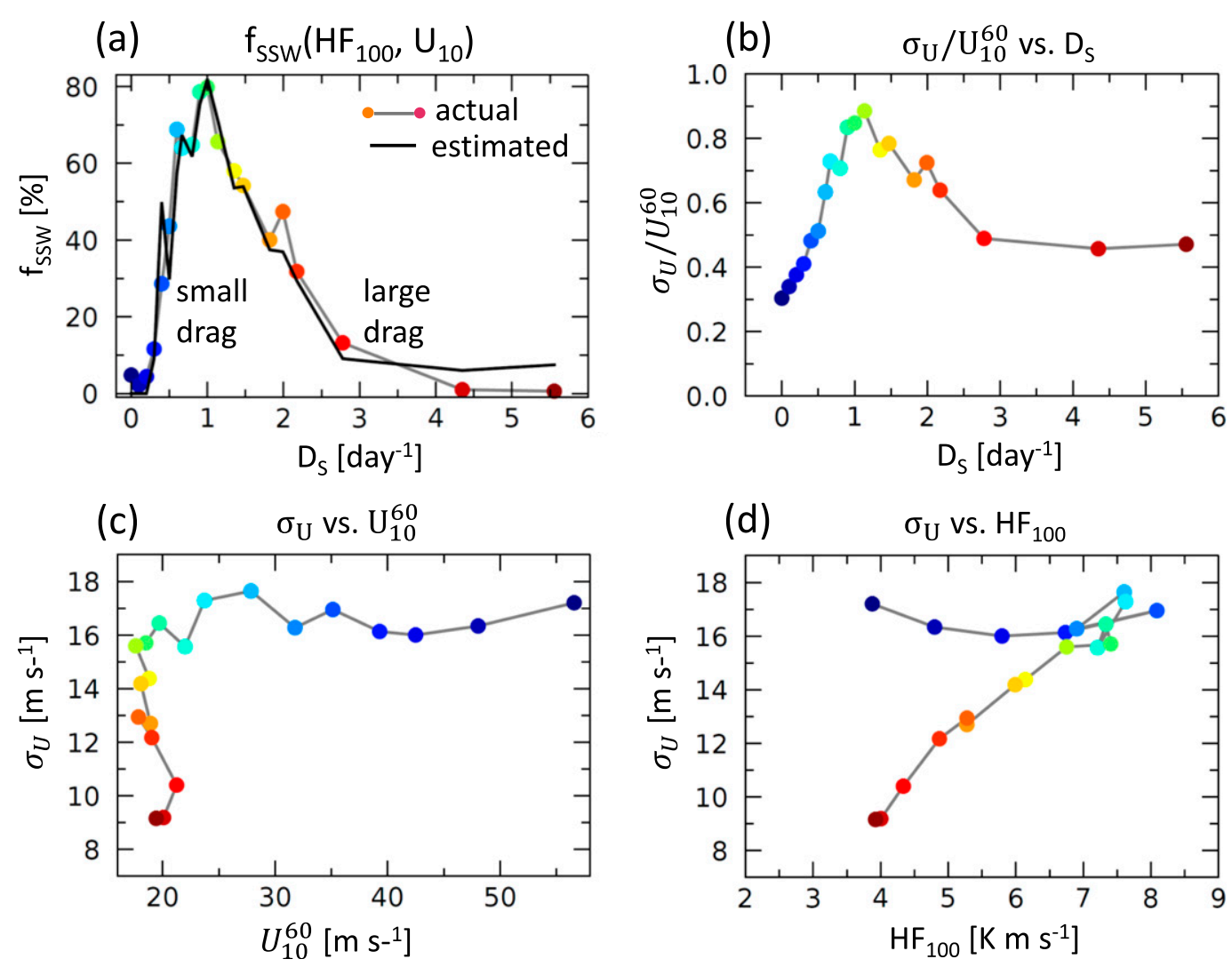

FIG. 1. (a) SSW frequency vs surface drag, (b) ratio between variability $\left(\sigma_{U}\right)$ and strength of zonal-mean zonal wind at $10 \mathrm{hPa}$ and $60^{\circ} \mathrm{N}\left(U_{10}^{60}\right)$ vs surface drag, (c) $\sigma_{U}$ vs $U_{10}^{60}$, and (d) $\sigma_{U}$ vs total heat fluxes at $100 \mathrm{hPa}\left(\mathrm{HF}_{100}\right)$. Colored markers are actual outcomes from the experiments, and the black line in (a) is estimated SSW frequency $\left(\hat{f}_{\text {SSW }}\right)$ from the regression model using $\mathrm{HF}_{100}$ and zonal-mean zonal wind at $10 \mathrm{hPa}\left(U_{10}\right)$ as predictors $\left(\hat{f}_{\mathrm{SSW}}=20.3 \mathrm{HF}_{100}-3.2 U_{10}-1.3, R^{2}=0.9\right)$.

zonal-mean zonal wind at $10 \mathrm{hPa}$ and $60^{\circ} \mathrm{N}\left(U_{10}^{60}\right)$. Two wind reversals are treated as distinct SSWs if they are apart by at least 20 days.

The synoptic variability (SVAR) of sea level pressure (SLP) over the northern extratropics is computed using the variance of a 24-h difference filter (Chang et al. 2012):

$$
\operatorname{SVAR}=\overline{[\operatorname{SLP}(t+24 \mathrm{~h})-\operatorname{SLP}(t)]^{2}},
$$

where the overbar represents time averaging over JFM. SVAR is first computed at each grid point, and then area averaged over all longitudes and from $30^{\circ}$ to $70^{\circ} \mathrm{N}$.

Following Hoskins and Valdes (1990), the maximum Eady growth rate, which is a measure of the baroclinic instability, is defined as

$$
\sigma_{\mathrm{BI}}=0.31 \frac{|f|}{N}\left|\frac{d u}{d z}\right|
$$

where $f$ is the Coriolis parameter and $N$ is the buoyancy frequency. Using monthly data, $\sigma_{\mathrm{BI}}$ is computed at the latitude of the maximum zonal-mean zonal wind at
$850 \mathrm{hPa}$, using differences in zonal-mean zonal wind (for $d u / d z$ ) and temperature (for $N$ ) at 850 and $250 \mathrm{hPa}$.

The meridional heat flux is calculated from daily $\left[v^{\prime} T^{\prime}\right]$, where primes denote deviations from the zonal mean and the brackets zonal averaging. The total heat flux is based on actual daily $v$ and $T$, and the stationary component is based on daily climatological $v$ and $T$. Climatological values are derived by averaging the daily data over JFM and (total only) all 500 years. The climatological transient component is the difference between the total heat flux and its stationary component.

We use the total climatological heat flux at $100 \mathrm{hPa}$ $\left(\mathrm{HF}_{100}\right)$ for the strength of the stratospheric wave driving, the maximum zonal-mean zonal wind at $10 \mathrm{hPa}$ $\left(U_{10}\right)$ for the strength of the stratospheric polar vortex, the zonal-mean zonal wind at the lowest model level $(925 \mathrm{hPa})$ and the same latitude as $U_{10}$ for the nearsurface wind $U_{S}$, and SVAR from (1) for the synoptic variability at the surface. We also tested an alternative definition for $U_{10}$, which is based solely on winters without SSWs. We did so to reduce influences of the 
SSW frequency, which we try to explain in this study, on the climatological vortex strength, which is one of our predictors for the SSW frequency. However, as we discuss later, using the alternative definition does not change our results. Since using all years is a simpler and cleaner way to create a climatology, we kept our original $U_{10}$ definition.

Finally, we use regression analysis and other mathematical models to explain variations in the occurrence frequency of SSWs $\left(f_{\mathrm{SSW}}\right)$ in terms of more fundamental dynamical quantities. The regressions reported in this study are all statistically significant at the $95 \%$ limit.

\section{Results}

Figure 1a shows how in our experiments the SSW frequency varies as a function of the surface drag. The relationship is nonlinear and nonmonotonic, with a maximum frequency at a drag of $\sim 1$ day $^{-1}$. The maximum motivates us to separate the experiments into a "large drag" group (reddish markers) with surface drag of greater than 1 day $^{-1}$ and a "small drag" group (blueish markers) with surface drag of less than 1 day $^{-1}$. We note that other separation criteria (e.g., low and high $f_{\mathrm{ssw}}$ ) could have been used, but as we will show later, our approach is meaningful as the small drag and large drag group show different dynamical behaviors. In what follows, we hope to better understand the interesting behavior of the SSW frequency with changing surface drag. We do so by using the outcomes from our experiments to derive empirical relationships between the quantities of interest and combining them with existing theoretical understanding.

\section{a. Frequency of SSWs}

As discussed in the introduction, the frequency of SSWs can be explained from the competing effects of variability and mean strength of the stratospheric polar vortex. For example, SSWs are more likely if the vortex is weaker (with all else being unchanged), since the detection of SSWs is based on the zonal-mean zonal wind to cross the absolute threshold of $0 \mathrm{~m} \mathrm{~s}^{-1}$. SSWs are also more likely if the variability of the polar vortex is higher, making it again more likely for the winds to cross the SSW threshold. In fact, Horan and Reichler (2017) demonstrated that a model's SSW frequency can be fairly well diagnosed from just knowing the strength and variability of the vortex. In other words, the ratio between variability and strength is roughly proportional to the SSW frequency, which can be seen by comparing this ratio (Fig. 1b) with the SSW frequency (Fig. 1a).

Martineau et al. (2018) suggested that the strength and variability of the vortex are related to each other, and that a weak lower stratospheric vortex tends to be associated with reduced vortex variability. For example, weak lower stratospheric winds may promote the formation of a critical layer and reduce the upward propagation of wave activity. We therefore investigate the relationship between the strength of the zonal-mean zonal wind at $60^{\circ} \mathrm{N}$ at various stratospheric levels and the daily standard deviation of the wind at $60^{\circ} \mathrm{N}$ and $10 \mathrm{hPa}\left(\sigma_{U}\right)$. Figure $1 \mathrm{c}$ shows the outcome for using the wind strength at $10 \mathrm{hPa}$ and $60^{\circ} \mathrm{N}\left(U_{10}^{60}\right)$. For the large drag experiments (reddish markers), $\sigma_{U}$ increases systematically while $U_{10}^{60}$ remains almost unchanged, whereas for the small drag experiments (blueish markers), $\sigma_{U}$ remains almost constant while $U_{10}^{60}$ increases strongly. Hence, there is no simple linear relationship between variability and strength, and besides vortex strength there must be additional influences affecting vortex variability. We also tested the wind strength at other levels $(100,50$, and $30 \mathrm{hPa})$ and found very similar relationships with $\sigma_{U}$ (not shown). This is in contrast to Martineau et al. (2018) and is probably related to different stratospheric temperature structures between the two studies.

Horan and Reichler (2017), Martineau et al. (2018), and Shaw et al. (2014) showed that the variability of the polar vortex is also related to the upward fluxes of wave activity at $100 \mathrm{hPa}\left(\mathrm{HF}_{100}\right)$, the so-called stratospheric wave driving. In Fig. 1d, we present this relationship. For the large drag experiments (reddish markers), the two quantities are nearly linearly related to each other. On the other hand, for the small drag experiments (blueish markers), variability remains high despite a decrease in heat flux. Figures 1c and $1 \mathrm{~d}$ combined suggest that the vortex variability is influenced by both vortex strength and stratospheric wave driving. For the large drag experiments, strength is constant, and variability increases because of the increase in wave driving. For the small drag experiments, variability is nearly constant because of the compensating effects of increasing strength (more variability) and decreasing wave driving (reduced variability).

We conclude that the decrease of the ratio (Fig. 1b) and of the SSW frequency with increasing drag for the large drag experiments (Fig. 1a) is associated with the heat flux reduction. For the small drag experiments, the decrease of the SSW frequency with declining drag is due to the combined effects of changing heat flux and $U_{10}$; this leads to an almost constant variability, but the increasing vortex strength reduces the ratio between variability and strength and thus the SSW frequency. We next use bilinear regression to model the SSW frequency using $\mathrm{HF}_{100}$ and $U_{10}$ as predictors. The regression model takes the form 

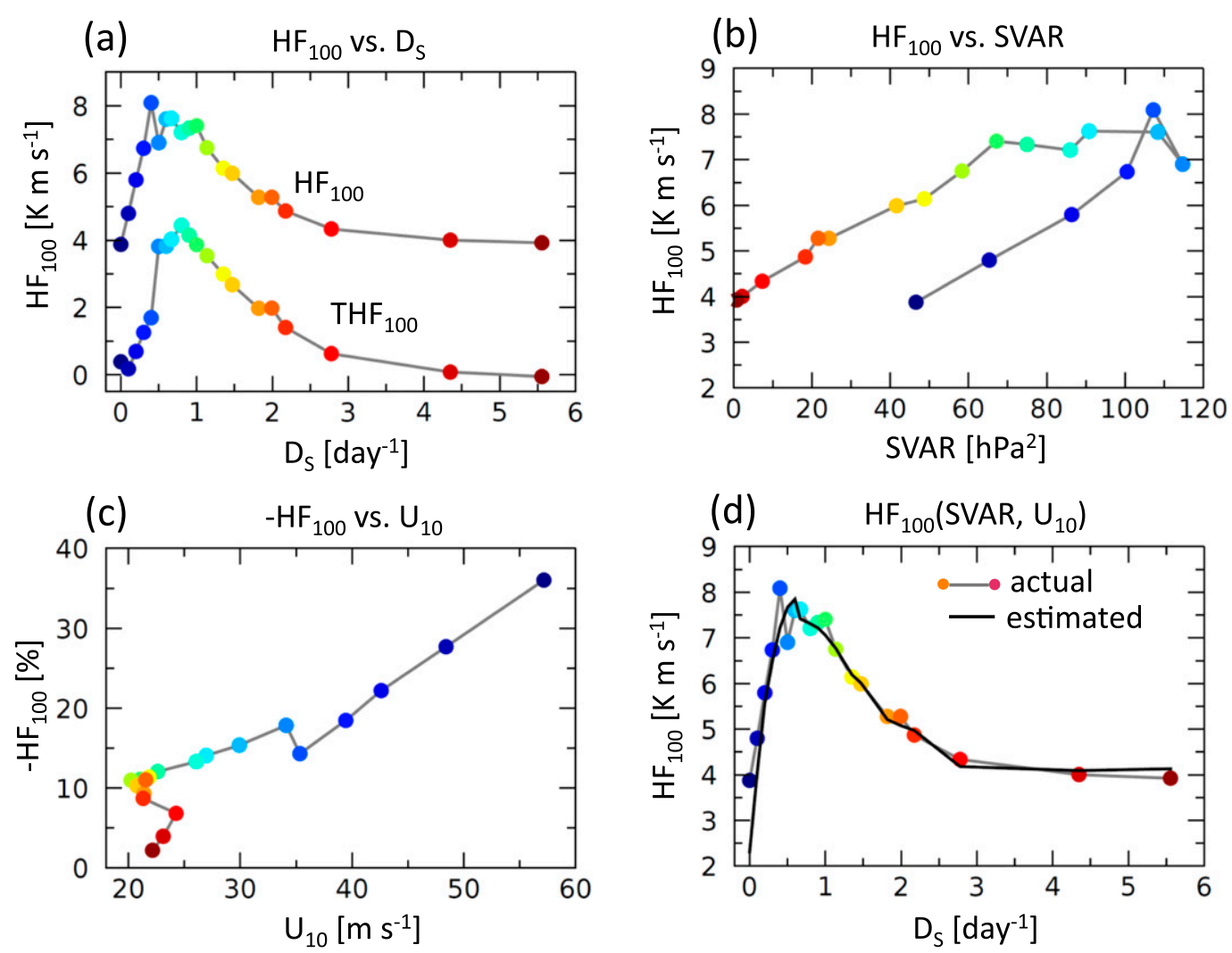

FIG. 2. Meridional heat fluxes at $100 \mathrm{hPa}$. (a) Total heat flux $\left(\mathrm{HF}_{100}\right)$ vs surface drag and its transient $\left(\mathrm{THF}_{100}\right)$ component, (b) total heat flux vs surface synoptic variability (SVAR), (c) percentage of days during JFM with a negative total heat flux vs climatological vortex strength $\left(U_{10}\right)$, and $(\mathrm{d})$ actual (markers) and estimated (black line) $\widehat{\mathrm{HF}}_{100}$ from the regression model using SVAR and $U_{10}$ as inputs $\left(\widehat{\mathrm{HF}}_{100}=0.04 \mathrm{SVAR}-0.1 U_{10}+6.5, R^{2}=0.94\right)$.

$$
\hat{f}_{\mathrm{SSW}}=a \mathrm{HF}_{100}+b U_{10}+c
$$

where $a, b$, and $c$ are chosen to minimize the misfit between the model and the actual $f_{\mathrm{Ssw}}$ values from the experiments. Our experiments are somewhat unevenly distributed in $D_{S}$ space, with a concentration of experiments with rather small $D_{S}$ values. Therefore, we consider here and in our subsequent analysis only a subset of 13 more evenly distributed experiments in determining regression parameters and calculating $R^{2}$ values. As shown by the black curve in Fig. 1a, the regression model recreates the actual $f_{\text {SSw }}$ (colored makers) quite well. Consistent with our expectation that increasing heat flux increases the SSW frequency and increasing vortex strength decreases the frequency, $a>0, b<0$, and $c \sim 0$ (see the caption of Fig. 1).

\section{b. Stratospheric wave driving}

Since the heat flux at $100 \mathrm{hPa}$ is important for the SSW frequency, we next study the variations of the heat flux as a function of the surface drag. As shown in Fig. 2a, the variations in the total heat flux $\left(\mathrm{HF}_{100}\right)$ are largely due to the variations in the transient heat flux $\left(\mathrm{THF}_{100}\right)$, as the stationary heat flux across the experiments (not shown) is mostly constant at about $4 \mathrm{~K} \mathrm{~m} \mathrm{~s}^{-1}$. The transient heat flux is due to the activity of transient eddies, which are generated in the troposphere through baroclinic instability (Chang et al. 2002; Shepherd and McLandress 2011; Limpasuvan and Hartmann 2000). We use the synoptic variability SVAR, the day-to-day variance of sea level pressure, as a proxy for the transient eddy activity and find that, to first order, the total heat flux exhibits a positive relationship with SVAR (Fig. 2b). This relationship is almost linear within the two groups of experiments. However, the two groups are somewhat offset from each other, such that for similar SVAR the large drag experiments (reddish markers) lead to more $\mathrm{HF}_{100}$ than the small drag experiments (blueish markers).

The difference between the large and small drag experiments indicates that the heat flux is not only influenced by the transient eddy activity. Another influence may be related to variations in the strength of the polar vortex. For example, Perlwitz and Graf (2001) found observational evidence for increased reflection of 
planetary waves from the stratosphere back into the troposphere (and hence reduced heat fluxes at $100 \mathrm{hPa}$ ) when the polar vortex in the lower stratosphere was strong. This is consistent with the theorem of Charney and Drazin (1961) that downward reflection occurs when the wind exceeds a certain critical value. On the other hand, Perlwitz and Harnik (2003) suggested that negative vertical wind shear above the jet core is an important condition for downward reflection. Such negative wind shear would require weak upper stratospheric winds (Shaw and Perlwitz 2014).

To understand how wave reflection and vortex strength relate to each other in our experiments, we analyze the percentage of days with a negative daily heat flux as an indicator for the likelihood of wave reflection. Figure $2 \mathrm{c}$ demonstrates that for the small drag experiments (blueish markers), there exists a fairly linear negative relationship between vortex strength $\left(U_{10}\right)$ and wave reflection. In additional analysis (not shown) we also find for the small drag experiments that a stronger climatological $U_{10}$ is associated with increased instances of daily negative wind shear in the upper stratosphere, in part confirming the ideas by Perlwitz and Harnik (2003). For the large drag experiments (reddish markers), however, Fig. $2 \mathrm{c}$ shows that the polar vortex is always weak $\left(20-25 \mathrm{~m} \mathrm{~s}^{-1}\right)$ and the percentage of days with wave reflection is small $(<10 \%)$. The particular split shape of the curve for $\mathrm{HF}_{100}$ versus SVAR (Fig. 2b) can therefore be interpreted as follows: beginning with the large drag experiments (reddish markers), heat flux increases almost linearly with the synoptic variability, as wave reflection is small (Fig. 2c); as the surface drag decreases to about 1 day $^{-1}$ (light green marker), wave reflection becomes more important, leading to a leveling off of heat flux with increasing synoptic variability (Fig. 2b); finally, as surface drag decreases further (blueish markers), both increasing wave reflection and decreases in synoptic variability reduce the heat flux again.

In summary, we find that change in total heat flux is related to a bottom-up mechanism from the synoptic tropospheric variability and to a top-down mechanism from wave reflection due to changes in the strength of the stratospheric polar vortex. As before, we use linear regression

$$
\widehat{\mathrm{HF}}_{100}=a \mathrm{SVAR}+b U_{10}+c,
$$

to test our assumptions (Fig. 2d). We find that, as expected, $a$ is positive, and that $b$ is negative (see caption Fig. 2), suggesting that in the climatological mean of our experiments a stronger polar vortex is related to reduced stratospheric wave driving, perhaps because of the increased percentage of negative heat flux days (Fig. 2c).

\section{c. Polar vortex strength}

The strength of the polar vortex, measured in terms of $U_{10}$, is another important factor for the SSW frequency. We now study how $U_{10}$ varies from experiment to experiment and try to understand what causes the variations. To this end, we decompose $U_{10}$ into a geostrophic component $U_{G}$ and an ageostrophic component $U_{A}$ at $10 \mathrm{hPa}$. The geostrophic component is given by the sum of the thermal wind $U_{T}$ and the geostrophic component of the near-surface wind $U_{S}$, so that

$$
U_{10}=U_{T}+U_{\mathrm{SG}}+U_{A},
$$

where $U_{\mathrm{SG}}$ is the geostrophic component of the surface wind, and $U_{A}$ is simply the residual between $U_{10}$ and $U_{G}$. Note that $U_{T}$ is calculated from the thermal wind relationship using the climatological mean temperatures. Figure 3 a presents the decomposition of $U_{10}$ for the different experiments. Both $U_{\mathrm{SG}}$ (green) and $U_{10}$ (black) vary in similar ways: they are about constant for larger drags and increase rapidly as the surface drag is reduced. The variations of $U_{T}$ (red) across the experiments are small because, by construction, all experiments have a very similar reanalysis-based temperature climatology. Both $U_{A}$ and its variations (blue) are also small, so that the variations of $U_{10}$ are dominated by $U_{\mathrm{SG}}$.

Upward propagating planetary waves and their breaking and dissipation also influence $U_{10}$. More specifically, $\mathrm{HF}_{100}$ impacts stratospheric temperatures and thus $U_{T}$, and to the extent that the polar vortex does not achieve geostrophic balance, $\mathrm{HF}_{100}$ also alters $U_{A}$. Since increasing $\mathrm{HF}_{100}$ decreases both $U_{T}$ and $U_{A}$, we expect a negative relationship between $\mathrm{HF}_{100}$ and the sum of $U_{T}$ and $U_{A}$. This is confirmed in Fig. 3b, but the negative relationship is weak $(r=-0.35)$. Figure 3 a shows that the variations of $U_{T}$ and $U_{A}$ largely oppose each other, such that the variations of $\left(U_{T}+U_{A}\right)$ are only a small residual. This helps explaining the noisy character of Fig. 3b. Further analysis (not shown) reveals that the relationship between $U_{T}\left(U_{A}\right)$ and $\mathrm{HF}_{100}$ is negative for the small (large) drag experiments and about zero for the large (small) drag experiments. Combined, this explains the overall negative correlation shown in Fig. 3b.

In summary, based on Fig. 3, we argue that variations in the strength of the polar vortex are largely related to variations in the near-surface wind, and that variations in the stratospheric wave driving also play some role. We test this using linear regression in the form of

$$
\hat{U}_{10}=a \mathrm{HF}_{100}+b U_{\mathrm{SG}}+c .
$$

As shown in the caption of Fig. 3 and as anticipated, $a$ is negative and $b$ is positive: $a=-0.7$, so that the first term 

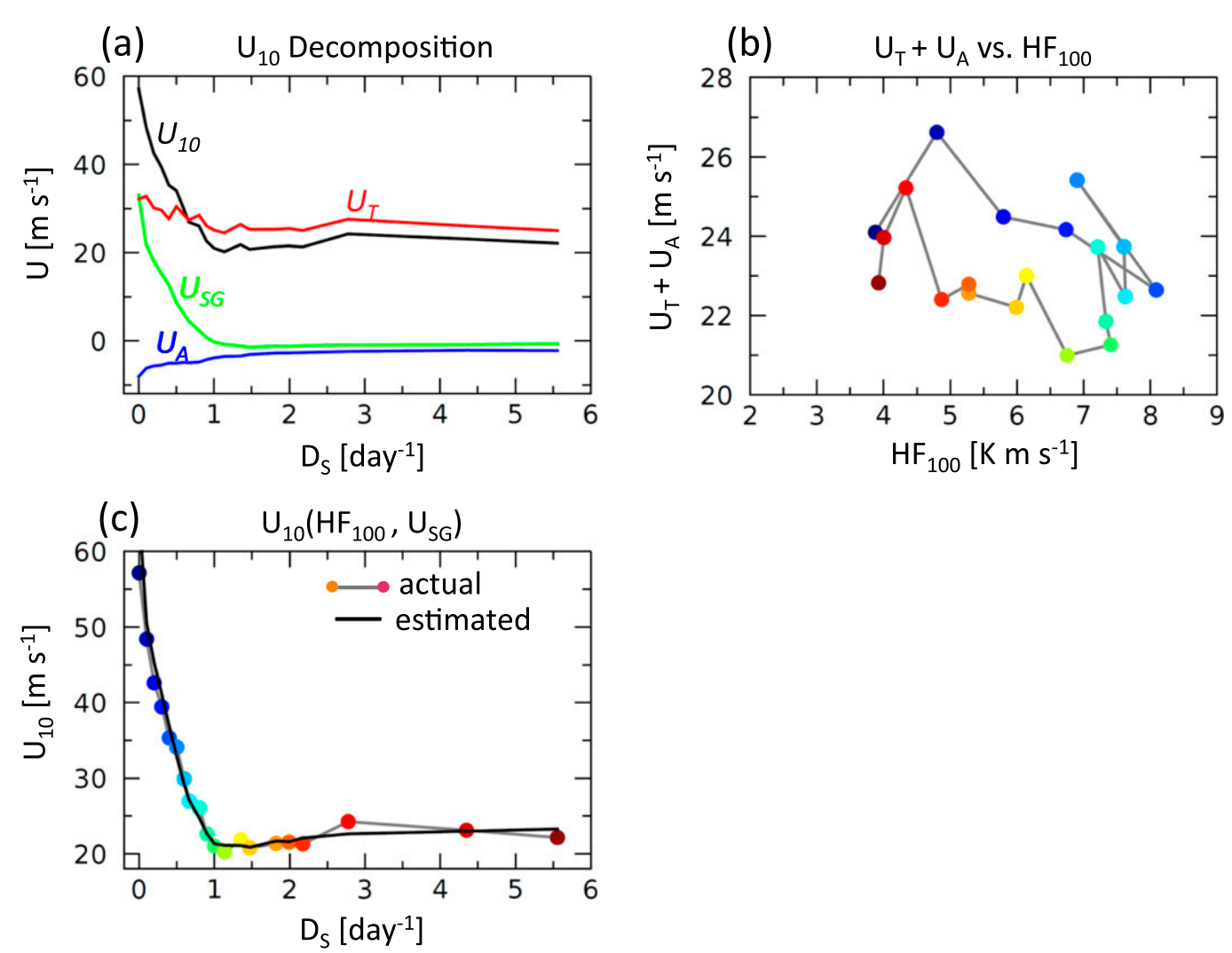

FIG. 3. Polar vortex strength $U_{10}$. (a) Decomposition of $U_{10}$ (black) into thermal wind $U_{T}$ (red), near-surface geostrophic wind $U_{S G}$ (green), and ageostrophic wind $U_{A}$ (blue); (b) $\left(U_{T}+U_{A}\right)$ vs $\mathrm{HF}_{100}$; and (c) actual $U_{10}$ (markers) and estimated $\hat{U}_{10}$ (black line) from regression using $\mathrm{HF}_{100}$ and $U_{S G}$ as inputs $\left(\hat{U}_{10}=-0.7 \mathrm{HF}_{100}+1.2 U_{\mathrm{SG}}+27\right.$, $\left.R^{2}=0.96\right)$.

contributes only little $(\sim 10 \%)$ to the overall variations of $U_{10}$, and $b=1.2$ is around 1 , as expected from (5). Also, $c=27 \mathrm{~m} \mathrm{~s}^{-1}$, which is close to the mean thermal wind $U_{T}$ shown in Fig. 3a. The outcome of the simple regression leads to an overall good fit $\left(R^{2}=0.96\right)$, as shown by the black curve in Fig. 3c. Replacing in (6) $U_{\mathrm{SG}}$ by $U_{S}$ leads to comparable outcomes since $U_{\mathrm{SG}}$ and $U_{S}$ vary in similar ways. In what follows, we therefore simply replace $U_{\mathrm{SG}}$ by $U_{S}$.

\section{d. The influence of surface drag}

The main goal of this study is to understand the influence of the surface drag $D_{S}$ on the frequency of SSWs (Fig. 1a). Since $f_{\mathrm{SSW}}, \mathrm{HF}_{100}$, and $U_{10}$ are all strongly related to SVAR and $U_{S}$, we investigate in a next step how SVAR and $U_{S}$ vary with $D_{S}$. We start with $U_{S}$ and find that it decreases approximately exponentially with $D_{S}$ (Fig. 4a). This motivates us to approximate $U_{S}$ by

$$
\hat{U}_{S}=a e^{-b D_{S}},
$$

where the parameters $a$ and $b$ are empirically determined by minimizing the misfit. The black curve in
Fig. 4a shows the outcome of (7), leading to a good fit with the actual $U_{S}$ from the experiments (colored markers).

The synoptic variability shows a more complicated relationship with the surface drag (Fig. 4b). SVAR maximizes at a $D_{S}$ value of $\sim 0.6 \mathrm{day}^{-1}$, and decreases from this maximum with either decreasing or increasing drag. The decrease with increasing drag is physically plausible since the drag suppresses the variability. Somewhat puzzling is the decrease of SVAR with decreasing surface drag. This behavior is similar to that of the SSW frequency (Fig. 1a) and the heat flux (Fig. 2a) with the surface drag, except that the peaks occur at different $D_{S}$ values. Understanding and describing the relationship between SVAR and $D_{S}$ is therefore important to resolving our problem. As we will explain in more detail below, we believe that the decrease of SVAR with decreasing surface drag is related to the socalled barotropic governor effect (James and Gray 1986; James 1987; Whitaker and Dole 1995), which describes the suppression of baroclinic instability with increasing meridional shear of the zonal flow. 

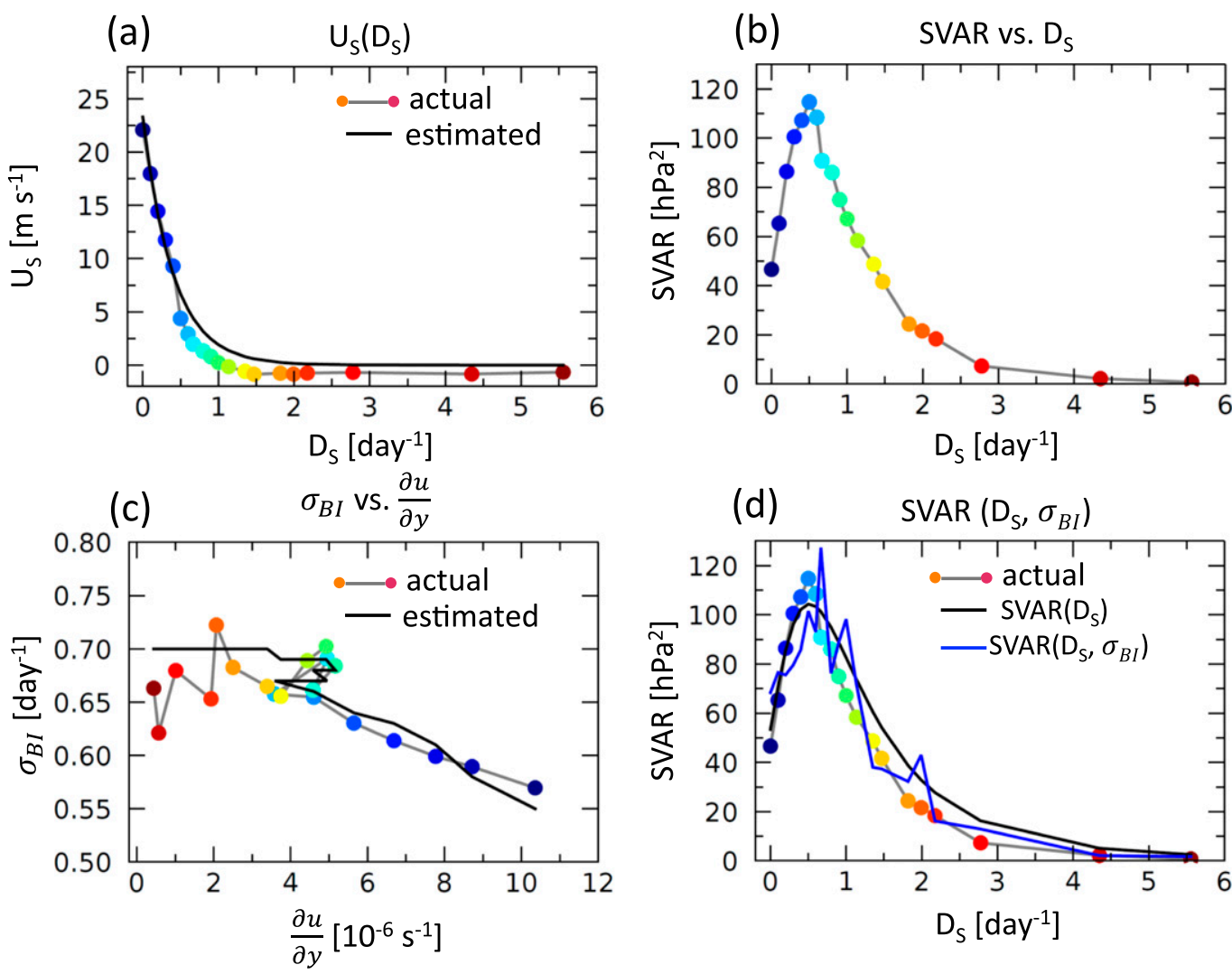

FIG. 4. Surface drag influences. (a) Near-surface zonal wind $\left(U_{S}\right)$ and its estimates $\left(\hat{U}_{S}=23.3 e^{-2.5 D_{S}}, R^{2}=0.73\right)$ and (b) synoptic variability (SVAR) as functions of surface drag. (c) Maximum Eady growth rate $\left(\hat{\sigma}_{\mathrm{BI}}=0.7-0.15 e^{-2.5 D_{s}}, R^{2}=0.50\right)$ as a function of the meridional shear $\partial u / \partial y$ of the 850 -hPa zonal wind $\left(U_{850}\right)$. Meridional shear is the difference between the maximum $U_{850}$ and $U_{850}$ at $20^{\circ}$ to the south of it. Markers show actual values and black lines are mathematical solutions. (d) SVAR as a function of surface drag and estimates of it based on (12) with $m=5 \mathrm{hPa}^{2}$. For calculating $\beta$ (see the appendix), (black) is based on an estimated Eady growth rate after (9) $\left(R^{2}=0.93\right)$, shown by the black curve in (c), and (blue) is based on the actual Eady growth rate $\left(R^{2}=\right.$ $0.89)$, determined from the experiment data and shown by the colored markers in (c).

We attempt to model mathematically the behavior of SVAR with changing surface drag. Remember, SVAR is the variance of daily sea level pressure, calculated at individual grid points, and then area averaged over the extratropics. The synoptic variability is closely related to the frequency and strength of extratropical storms. Theory by Eady (1949) describes how meridional temperature gradients and associated vertical wind shear work together with vertical stability in amplifying such storms in a process known as baroclinic instability. But the surface drag $D_{S}$ also influences SVAR, as $D_{S}$ slows the growth of the eddies. Thus, both baroclinic instability and momentum damping in the boundary layer are important for SVAR. We use the damped harmonic oscillator model

$$
\frac{d^{2} x}{d t^{2}}+f_{d} D_{s} \frac{d x}{d t}-\sigma_{\mathrm{BI}}^{2} x=0
$$

to combine these two influences into one model. Here, the displacement of unitless quantity $x$ is analogous to a change in sea level pressure at some grid point; the scaling factor $f_{d}=0.3$ takes into account of the decrease of the surface drag with height, so that its product with $D_{S}$ creates a vertically representative drag. Note the minus sign in front of the third term, leading to exponentially growing solutions. According to (2), $\sigma_{\mathrm{BI}}$ is the maximum growth rate of eddies, empirically determined from our experiments.

The colored markers in Fig. 4c show the maximum Eady growth rate derived from our experiments based on (2) at the latitude of the maximum zonal wind at $850 \mathrm{hPa}$. For the large drag experiments (reddish markers), the growth rate fluctuates around $0.7 \mathrm{day}^{-1}$. For the small drag experiments (blueish markers), however, the growth rate systematically decreases from larger to smaller drag. At the same time, Fig. 4c indicates that the meridional wind shear at $850 \mathrm{hPa}$, a measure for the barotropic governor effect, systematically increases from larger to smaller drag. This suggests that the 
decrease of the growth rate with decreasing drag is related to the suppression of instability by the barotropic governor effect. Indeed, for the small drag experiments there exists an almost linear relationship between growth rate and wind shear and thus the governor effect (not shown). As the wind shear shows an exponential relationship with the surface drag (not shown), we estimate the Eady growth rate by

$$
\hat{\sigma}_{\mathrm{BI}}=c-a e^{-b D_{s}},
$$

where the parameter $b$ is the same as in (7), as the wind shear scales linearly with $U_{850}$ and $U_{S} ; a$ and $c$ are empirically determined. The black curve in Fig. $4 \mathrm{c}$ shows the outcome of our estimate for $\sigma_{\mathrm{BI}}$ using (9). Later in this paper, we will employ both the empirical and estimated Eady growth rates to determine SVAR and the SSW frequency.

Figure $4 \mathrm{~b}$ suggests that SVAR and thus the behavior of $x$ in (8) are determined by the joint effects of damping and instability, which have different contributions in our two groups of experiments: with increasing drag, the momentum damping term dominates the solution of $x$ and leads to a weakening of eddy activity and SVAR; with decreasing drag, the decrease in the Eady growth rate because of the barotropic governor effect becomes important in reducing the growth of baroclinic eddies.

We next try to quantify these ideas and use (8) to find an expression for the variations of SVAR with surface drag. In analogy to (1), we use

$$
\widehat{\operatorname{SVAR}} \propto \Delta x^{2}=\left(x_{t+1}-x_{t}\right)^{2}
$$

to estimate SVAR for some arbitrary day $t$, where $\Delta x^{2}$ represents the squared difference in sea level pressure between two subsequent days. Since according to (8) $x$ increases with increasing $\sigma_{\mathrm{BI}}$ or decreasing $D_{S}, \Delta x$ is also a function of $D_{S}$ and $\sigma_{\mathrm{BI}}$. As explained in the appendix, $\Delta x$ is given by

$$
\Delta x \cong A_{2}(\beta-\alpha) e^{(\beta-\alpha)[t+(1 / 2)]},
$$

where the vertically averaged momentum damping $\alpha$ is a linear function of $D_{S}, \beta$ is a function of both $D_{S}$ and $\sigma_{\mathrm{BI}}$, and $(\beta-\alpha)$ is the growth rate of $x$. More details are given in the appendix. Inspection of (11) shows that $\Delta x$ goes to zero for large $D_{S}$ and to some finite positive value (determined by $\sigma_{\mathrm{BI}}$ ) for very small $D_{S}$. In other words, large drag suppresses the growth of eddies. In contrast, if the drag becomes smaller, growth related to $\sigma_{\mathrm{BI}}$ becomes increasingly important. Since we are only interested in the variations of SVAR across the different experiments, and since (8) only describes the growth of eddies and not their decay, we do not attempt to

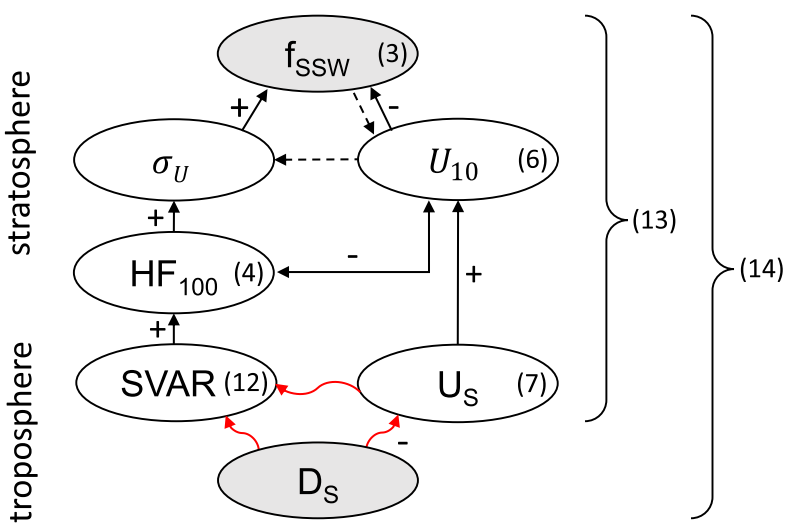

FIG. 5. Conceptual view of how the surface drag influences the frequency of SSWs. Indicated are the direction and sign of influence between various key dynamical quantities of the stratosphere and troposphere. Numbers in parentheses indicate corresponding equations. Black straight arrows indicate linear relationships, red curved arrows indicate nonlinear relationships, and dashed arrows indicate possible influences that are not included in this study.

calculate a mean $\Delta x^{2}$ over many days as in (1) but instead simply chose a representative $\Delta x^{2}$ for $t=4$ days. This is a reasonable choice, given the several-day-long life cycle of baroclinic eddies. Then, SVAR is estimated from

$$
\widehat{\mathrm{SVAR}}=m \Delta x_{t=4}^{2},
$$

where $m$ is derived by regression. We note that choosing a different $t$ would have only impacted $m$, but not our estimate of SVAR. The curves in Fig. $4 d$ show the estimated SVAR based on (12). The blue curve is based on the actual Eady growth rate from the experiment data as inputs. The black curve is based on an estimated Eady growth rate using (9) and contains only information about $D_{S}$. Both curves show good agreement with the actual data.

\section{e. The final model}

We now summarize our ideas in terms of a schematic diagram, showing how, to first order, the surface drag influences the frequency of SSWs (Fig. 5). According to (3), both the heat fluxes at $100 \mathrm{hPa}\left(\mathrm{HF}_{100}\right)$ and the strength of the polar vortex $\left(U_{10}\right)$ influence the frequency of SSWs $\left(f_{\mathrm{SSW}}\right)$ : increasing heat fluxes increase the number of SSWs, while increasing vortex strength decreases the number of SSWs. The heat fluxes are positively related to the tropospheric synoptic variability (SVAR) and negatively influenced by the polar vortex strength through wave reflection. On the other hand, the polar vortex is strengthened by the surface wind $\left(U_{S}\right)$ and weakened by the heat fluxes. Thus, the heat fluxes are under a negative influence of the 

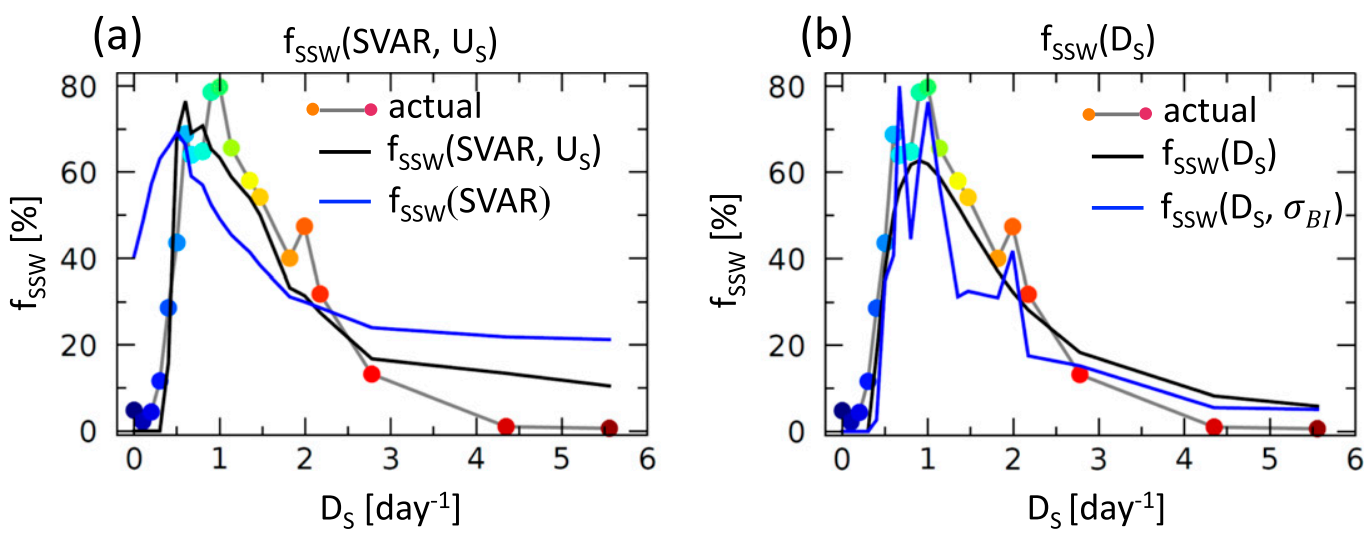

FIG. 6. Estimated SSW frequency, (a) based on (13), with (black) using SVAR and $U_{S}$ as inputs $\left(\hat{f}_{\text {SSW }}=\right.$ $\left.0.92 \mathrm{SVAR}-9.3 U_{S}+3.5, R^{2}=0.81\right)$ and (blue) using only SVAR as input $\left(\hat{f}_{\mathrm{SSW}}=0.42 \mathrm{SVAR}+20.9, R^{2}=0.41\right)$ and (b) based on (14), with (blue) using $D_{S}$ and $\sigma_{\mathrm{BI}}$ as inputs $\left(R^{2}=0.66\right)$ and (black) using only $D_{S}$ as input $\left(\hat{f}_{\mathrm{SSW}}=4.6 \Delta x_{t=4}^{2}-217.6 e^{-2.5 D_{S}}+3.5, R^{2}=0.86\right)$.

surface wind, and the polar vortex strength is negatively influenced by the synoptic variability. Therefore, the SSW frequency is a function of synoptic variability and surface winds, with positive and negative correlations, respectively. The strength of the synoptic variability and the surface winds are both controlled by the surface drag $\left(D_{S}\right)$. There is a direct influence of the drag on the synoptic variability through the damping (8). As indicated by the red arrow from $U_{S}$ to SVAR, there is also an indirect effect of $D_{S}$ on SVAR, involving the barotropic governor effect and its influence by $U_{S}$. Overall, this provides an explanation for how in our experiments the surface drag influences the frequency of SSWs.

Since (3), (4), and (6) are all linear, we take the linear approach one step further to create a regression model for $f_{\mathrm{SSW}}$ based on SVAR and $U_{S}$ as inputs:

$$
\hat{f}_{\mathrm{SSW}}=d \mathrm{SVAR}+g U_{S}+f .
$$

The black line in Fig. 6a shows that this leads to an acceptable fit to the actual $f_{\mathrm{ssw}}$, with an explained variance of $81 \%$. As expected, $d$ is positive, $g$ is negative, and the constant $f$ is small. We also tested whether $U_{S}$ in (13) contains useful information about $f_{\mathrm{Ssw}}$, or whether SVAR alone is sufficient to estimate $f_{\text {Ssw. }}$. The blue curve in Fig. 6a shows $f_{\text {SSw }}$ from just using SVAR in (13). The explained variance is only $41 \%$, indicating that even though SVAR has a similar shape as $f_{\mathrm{sSW}}$, SVAR alone is not as good in explaining $f_{\text {Ssw. }}$. In other words, the nearsurface winds also play a role for $f_{\text {Ssw }}$ by reducing the SSW frequency for the small drag experiments and by shifting the frequency maximum to the right.

We next substitute SVAR from (12) and $U_{S}$ from (7) into (13) to arrive at

$$
\hat{f}_{\mathrm{SSW}}=d m \Delta x_{t=4}^{2}+g a e^{-b D_{S}}+f .
$$

The outcome is shown in Fig. 6b. The term $\Delta x$ is a function of $D_{S}$ and $\sigma_{\mathrm{BI}}$. The blue curve uses the actual $\sigma_{\mathrm{BI}}$ from the experiments, while the black curve is based only on $D_{S}$, using estimated $\sigma_{\mathrm{BI}}$ according to (9). Both approaches reproduce the variation of the SSW frequency across the experiments. Since $D_{S}$ is the only independent variable in (14), this accomplishes our original goal to give a dynamically consistent explanation for the influence of the surface drag on SSW frequency.

\section{Conclusions and discussion}

Based on the idealized model of WR18, we used a parameter sweep to study how the surface drag influences the frequency of SSWs. We found that the SSW frequency is a nonlinear function of the surface drag (Fig. 1a). The main goal of this study was to explain this relationship. As schematically summarized in Fig. 5, this was accomplished by using dynamical arguments that connect variations in the SSW frequency to a chain of relevant quantities and to the surface drag. Results from the sweep were used to quantify how individual elements of the chain are linked with each other. The final outcome was an empirical relationship that explains the nonlinear variations in SSW frequency from variations in the surface drag.

The basic assumption of our study was that the frequency of SSWs is mainly determined by the strength of the stratospheric polar vortex and the wave activity flux entering the stratosphere from below. We found that an increasing vortex strength decreases the frequency, and 
that an increasing wave activity increases it. Regarding the vortex strength, one could argue that information about the SSW frequency is contained in the climatological vortex strength because of the impact of SSWs on the vortex. Therefore, the strength may not be an independent predictor for the frequency. To address this possibility, we attempted to withhold information about SSWs from the vortex strength by forming an alternate zonal-mean zonal wind climatology, $U_{10}^{/ S \mathrm{SW}}$, which only includes years without SSWs. As expected, $U_{10}^{\text {SSW }}$ is stronger than $U_{10}$ (by $2-10 \mathrm{~m} \mathrm{~s}^{-1}$ ), but the functional relationship of $U_{10}^{\text {/SSW }}$ with other quantities is very similar to that of $U_{10}$. More precisely, using $U_{10}^{/ S S W}$ instead of $U_{10}$ in (3) leads to a very similar regression model, except that the explained variance is somewhat reduced $(0.9 \mathrm{vs}$ 0.8 ). We conclude that the vortex strength contains distinct information about the likelihood of SSWs that goes beyond the direct impact of SSWs.

Regarding the wave activity flux, Martineau et al. (2018) pointed out that it is not necessarily the timemean flux and the drag exerted by it on the polar vortex that determine the stratospheric variability and hence SSWs. Instead, they found that the distribution of the wave activity flux is important, with a broader distribution leading to more frequent bursts of extreme wave activity and more SSWs. In our study, the mean strength of wave activity is closely related to the width of its distribution and the probability of extreme positive heat flux events. This explains why, in our case, mean wave activity is a good predictor for stratospheric variability.

The synoptic eddy activity was the key dynamical quantity that helped us to relate the surface drag to the frequency of SSWs. This is because the synoptic eddies largely determine the transient waves, which in the real atmosphere contribute about half of the planetary wave activity entering the stratosphere (WR18). Variations in synoptic eddy activity are due to baroclinic instability on one hand and the stabilizing influence of the surface damping on the other hand. Critically, when the drag is small and unable to balance an otherwise growing instability, the barotropic governor effect (James 1987) becomes important in reducing the baroclinic instability. The synoptic eddy activity and its nonlinear dependence on drag, instability, and barotropic governor determine the characteristic relationship between SSW frequency and drag. A second nonlinear influence comes into play from drag-related variations in the surface wind, which, by the thermal wind relationship, influence the strength of the polar vortex.

This study is the first to systematically investigate influences on the climatological frequency of SSWs and in particular the impact of the surface drag on it. A potential caveat of our study is that not only the surface drag but also the equilibrium temperatures and thus the diabatic heating $Q$ vary across the experiments. Therefore, additional influences from $Q$ cannot be entirely ruled out. However, we find that the relative variations in $Q$ are small compared to those in surface drag and other dynamical quantities, suggesting that the overall influence of $Q$ on our results is small.

From the regime behavior of our experiments one may ask in which of the two regimes the actual atmosphere is. The $\sim 60 \%$ observed SSW frequency together with Fig. 1a suggest that the more realistic experiments are close to the SSW maximum and these experiments have $D_{S}$ values of $\sim 1$ day $^{-1}$. Interestingly, a $D_{S}$ value of one was also chosen by Held and Suarez (1994). Comparing the climate of our experiments against the reanalysis (as in WR18) we find that $D_{S}$ values of 0.9 $1.5 \mathrm{day}^{-1}$ are the optimal choice, suggesting that the real atmosphere belongs to the large drag regime and that the barotropic governor effect is not important.

Returning to our original question from the introduction of why climate models have such a large spread in their SSW frequency, we believe that our study can provide some potential answers. First, our results demonstrate that the SSW frequency depends on various stratospheric and tropospheric aspects of climate. As such, the frequency can be seen as a sensitive and integrative measure of model performance. Second, our study points to the importance of the stratospheric wave driving in generating a realistic SSW frequency. Our experiments had very similar stationary wave characteristics, and differed mostly in terms of the transient planetary waves. But full climate models are unlikely to behave like this, with varying transient and stationary wave components. In other words, climate models have an added degree of freedom in terms of the total planetary wave activity, which helps to explain the wide range of simulated SSW frequencies. Third, our study demonstrates that surface effects influence the stratosphere. As a corollary, the planetary boundary layer is important for the SSW frequency of a climate model. It still remains to be seen to what extent these and other model features are connected to the frequency of SSWs, an issue that we are currently investigating.

Acknowledgments. We thank the three anonymous reviewers for their comments and suggestions. We thank the National Science Foundation (NSF) for providing support for this research under Grant 1446292. We also thank the Department of the Atmospheric Sciences at the University of Utah for its support. A grant of computer time from the Center for High Performance Computing at the University of Utah is gratefully 
acknowledged. We also acknowledge the use of computational resources at the NCAR-Wyoming Supercomputing Center provided by NSF and the State of Wyoming, and supported by NCAR's Computational and Information Systems Laboratory. We thank NASA for providing the MERRA-2 reanalysis.

\section{APPENDIX}

\section{Solution of the Damped Harmonic Oscillator}

To solve the damped harmonic oscillator system (8), we assume an exponential solution in time

$$
x=A e^{\omega t},
$$

with growth rate $\omega$. Substituting (A1) into (8), we find that

$$
x(t)=A_{1} e^{-(\beta+\alpha) t}+A_{2} e^{(\beta-\alpha) t},
$$

where

$$
\begin{aligned}
\alpha & =\frac{f_{d} D_{S}}{2}, \\
\beta & =\sqrt{\frac{1}{4}\left(f_{d} D_{S}\right)^{2}+\sigma_{\mathrm{BI}}^{2}} .
\end{aligned}
$$

At $t=0$, we start from the arbitrary initial condition $x(0)=1$ and a state of rest $\dot{x}(0)=0$. Solving for $A_{1}$ and $A_{2}$, we obtain

$$
A_{1}=\frac{\beta-\alpha}{2 \beta} \text { and } A_{2}=\frac{\beta+\alpha}{2 \beta} .
$$

Since $\sigma_{\mathrm{BI}}^{2}$ is positive, $\beta$ is real and larger than $\alpha$, indicating that the system (A2) is growing. To diagnose SVAR, we substitute (A2)-(A5) into (10) and get

$$
\Delta x=x_{t+1}-x_{t}=\Delta x_{1}+\Delta x_{2},
$$

where

$$
\begin{aligned}
& \Delta x_{1}=A_{1}\left[e^{-(\beta+\alpha)(t+1)}-e^{-(\beta+\alpha) t}\right] \quad \text { and } \\
& \Delta x_{2}=A_{2}\left[e^{(\beta-\alpha)(t+1)}-e^{(\beta-\alpha) t}\right] .
\end{aligned}
$$

The terms $\alpha$ and $\beta$ are both real and positive, and $\beta$ is larger than $\alpha$. Thus, $\Delta x_{1}$ is exponentially decaying and $\Delta x_{2}$ is exponentially growing. It can be shown that when $t \geq 2$ days, $\Delta x_{1}$ is very small and can be neglected. Therefore, when $t \geq 2$ days, we can simplify $\Delta x \cong \Delta x_{2}$. We use the derivative at the midpoint between $t$ and $t+1$ to approximate $\Delta x_{2}$ as

$$
\Delta x \cong A_{2}(\beta-\alpha) e^{(\beta-\alpha)[t+(1 / 2)]} .
$$

\section{REFERENCES}

Baldwin, M. P., and T. J. Dunkerton, 2001: Stratospheric harbingers of anomalous weather regimes. Science, 294, 581-584, https://doi.org/10.1126/science.1063315.

Bosilovich, M. G., R. Lucchesi, and M. Suarez, 2016: MERRA-2: File specification. GMAO Office Note 9, 73 pp., https:// gmao.gsfc.nasa.gov/pubs/docs/Bosilovich785.pdf.

Chang, E. K. M., 2006: An idealized nonlinear model of the Northern Hemisphere winter storm tracks. J. Atmos. Sci., 63, 1818-1839, https://doi.org/10.1175/JAS3726.1.

, S. Lee, and K. L. Swanson, 2002: Storm track dynamics. J. Climate, 15, 2163-2183, https://doi.org/10.1175/15200442(2002)015<02163:STD>2.0.CO;2.

, Y. Guo, and X. Xia, 2012: CMIP5 multimodel ensemble projection of storm track change under global warming. J. Geophys. Res., 117, D23118, https://doi.org/10.1029/ 2012JD018578.

Charlton, A. J., and L. M. Polvani, 2007: A new look at stratospheric sudden warmings. Part I: Climatology and modeling benchmarks. J. Climate, 20, 449-469, https://doi.org/10.1175/ JCLI3996.1.

— , and Coauthors, 2007: A new look at stratospheric sudden warmings. Part II: Evaluation of numerical model simulations. J. Climate, 20, 470-488, https://doi.org/10.1175/JCLI3994.1.

Charlton-Perez, A. J., and Coauthors, 2013: On the lack of stratospheric dynamical variability in low-top versions of the CMIP5 models. J. Geophys. Res. Atmos., 118, 2494-2505, https://doi.org/10.1002/jgrd.50125.

Charney, J. G., and P. G. Drazin, 1961: Propagation of planetaryscale disturbances from the lower into the upper atmosphere. J. Geophys. Res., 66, 83-109, https://doi.org/10.1029/ JZ066i001p00083.

Chen, G., I. M. Held, and W. Robinson, 2007: Sensitivity of the latitude of the surface westerlies to surface friction. J. Atmos. Sci., 64, 2899-2915, https://doi.org/10.1175/JAS3995.1.

Coy, L., S. Eckermann, and K. Hoppel, 2009: Planetary wave breaking and tropospheric forcing as seen in the stratospheric sudden warming of 2006. J. Atmos. Sci., 66, 495-507, https:// doi.org/10.1175/2008JAS2784.1.

de la Cámara, A., J. R. Albers, T. Birner, R. R. Garcia, P. Hitchcock, D. E. Kinnison, and A. K. Smith, 2017: Sensitivity of sudden stratospheric warmings to previous stratospheric conditions. J. Atmos. Sci., 74, 2857-2877, https:// doi.org/10.1175/JAS-D-17-0136.1.

Eady, E. T., 1949: Long waves and cyclone waves. Tellus, 1, 33-52, https://doi.org/10.3402/tellusa.v1i3.8507.

Gerber, E. P., and L. M. Polvani, 2009: Stratosphere-troposphere coupling in a relatively simple AGCM: The importance of stratospheric variability. J. Climate, 22, 1920-1933, https:// doi.org/10.1175/2008JCLI2548.1.

Held, I. M., and M. M. J. Suarez, 1994: A proposal for the intercomparison of the dynamical cores of atmospheric general circulation models. Bull. Amer. Meteor. Soc., 75, 1825-1830, https://doi.org/10.1175/1520-0477(1994)075<1825: APFTIO $>2.0 . \mathrm{CO} ; 2$.

Hitchcock, P., and T. G. Shepherd, 2013: Zonal-mean dynamics of extended recoveries from stratospheric sudden warmings. J. Atmos. Sci., 70, 688-707, https://doi.org/10.1175/JAS-D-12-0111.1. 
Horan, M. F., and T. Reichler, 2017: Modeling seasonal sudden stratospheric warming climatology based on polar vortex statistics. J. Climate, 30, 10 101-10 116, https://doi.org/10.1175/ JCLI-D-17-0257.1.

Hoskins, B. J., and P. J. Valdes, 1990: On the existence of storm tracks. J. Atmos. Sci., 47, 1854-1864, https://doi.org/10.1175/ 1520-0469(1990)047<1854:OTEOST>2.0.CO;2.

James, I. N., 1987: Suppression of baroclinic instability in horizontally sheared flows. J. Atmos. Sci., 44, 3710-3720, https://doi.org/ 10.1175/1520-0469(1987)044<3710:SOBIIH > 2.0.CO;2.

, and L. J. Gray, 1986: Concerning the effect of surface drag on the circulation of a baroclinic planetary atmosphere. Quart. J. Roy. Meteor. Soc., 112, 1231-1250, https://doi.org/10.1002/ qj. 49711247417.

Jucker, M., 2016: Are sudden stratospheric warmings generic? Insights from an idealized GCM. J. Atmos. Sci., 73, 5061-5080, https://doi.org/10.1175/JAS-D-15-0353.1.

_, S. Fueglistaler, and G. K. Vallis, 2014: Stratospheric sudden warmings in an idealized GCM. J. Geophys. Res., 119, 11054 11 064, https://doi.org/10.1002/2014JD022170.

Lee, Y.-Y., and R. X. Black, 2015: The structure and dynamics of the stratospheric northern annular mode in CMIP5 simulations. J. Climate, 28, 86-107, https://doi.org/10.1175/JCLID-13-00570.1.

Limpasuvan, V., and D. L. Hartmann, 2000: Wave-maintained annular modes of climate variability. J. Climate, 13, 4414-4429, https://doi.org/10.1175/1520-0442(2000)013<4414:WMAMOC $>2.0 . \mathrm{CO} ; 2$.

— D. W. J. Thompson, and D. L. Hartmann, 2004: The life cycle of the Northern Hemisphere sudden stratospheric warmings. J. Climate, 17, 2584-2596, https://doi.org/10.1175/15200442(2004)017<2584:TLCOTN >2.0.CO;2.

Lindgren, E. A., A. Sheshadri, and R. A. Plumb, 2018: Sudden stratospheric warming formation in an idealized general circulation model using three types of tropospheric forcing. J. Geophys. Res. Atmos., 123, $10125-10139$, https://doi.org/ 10.1029/2018JD028537.

Martineau, P., G. Chen, S. Son, and J. Kim, 2018: Lower-stratospheric control of the frequency of sudden stratospheric warming events. J. Geophys. Res. Atmos., 123, 3051-3070, https://doi.org/10.1002/2017JD027648.

Matsuno, T., 1971: A dynamical model of the stratospheric sudden warming. J. Atmos. Sci., 28, 1479-1494, https://doi.org/10.1175/ 1520-0469(1971)028<1479:ADMOTS > 2.0.CO;2.

McInturff, R. M., Ed., 1978: Stratospheric warmings: Synoptic, dynamic and general-circulation aspects. NASA Reference Publ. NASA-RP-1017, 174 pp., http://ntrs.nasa.gov/archive/ nasa/casi.ntrs.nasa.gov/19780010687.pdf.

McIntyre, M. E., 1982: How well do we understand the dynamics of stratospheric warmings? J. Meteor. Soc. Japan, 60, 37-65, https://doi.org/10.2151/jmsj1965.60.1_37.

Perlwitz, J., and H.-F. Graf, 2001: Troposphere-stratosphere dynamic coupling under strong and weak polar vortex conditions. Geophys. Res. Lett., 28, 271-274, https://doi.org/ 10.1029/2000GL012405.

- and N. Harnik, 2003: Observational evidence of a stratospheric influence on the troposphere by planetary wave re- flection. J. Climate, 16, 3011-3026, https://doi.org/10.1175/ 1520-0442(2003)016<3011:OEOASI >2.0.CO;2.

Polvani, L. M., and D. W. Waugh, 2004: Upward wave activity flux as a precursor to extreme stratospheric events and subsequent anomalous surface weather regimes. J. Climate, 17, 3548-3554, https://doi.org/10.1175/1520-0442(2004)017<3548: UWAFAA $>2.0 . \mathrm{CO} ; 2$.

Richter, J. H., F. Sassi, and R. R. Garcia, 2010: Toward a physically based gravity wave source parameterization in a general circulation model. J. Atmos. Sci., 67, 136-156, https://doi.org/ 10.1175/2009JAS3112.1.

Scott, R. K., and L. M. Polvani, 2006: Internal variability of the winter stratosphere. Part I: Time-independent forcing. J. Atmos. Sci., 63, 2758-2776, https://doi.org/10.1175/JAS3797.1.

Shaw, T. A., and J. Perlwitz, 2010: The impact of stratospheric model configuration on planetary-scale waves in Northern Hemisphere winter. J. Climate, 23, 3369-3389, https://doi.org/ 10.1175/2010JCLI3438.1.

, and - 2014: On the control of the residual circulation and stratospheric temperatures in the Arctic by planetary wave coupling. J. Atmos. Sci., 71, 195-206, https://doi.org/10.1175/ JAS-D-13-0138.1.

, _ _ and O. Weiner, 2014: Troposphere-stratosphere coupling: Links to North Atlantic weather and climate, including their representation in CMIP5 models. J. Geophys. Res. Atmos., 119, 5864-5880, https://doi.org/10.1002/2013JD021191.

Shepherd, T. G., and C. McLandress, 2011: A robust mechanism for strengthening of the Brewer-Dobson circulation in response to climate change: Critical-layer control of subtropical wave breaking. J. Atmos. Sci., 68, 784-797, https://doi.org/ 10.1175/2010JAS3608.1.

Sheshadri, A., R. A. Plumb, and E. P. Gerber, 2015: Seasonal variability of the polar stratospheric vortex in an idealized AGCM with varying tropospheric wave forcing. J. Atmos. Sci., 72, 2248-2266, https://doi.org/10.1175/JAS-D-14-0191.1.

SPARC, 2010: SPARC CCMVal Report on the Evaluation of Chemistry-Climate Models. V. Eyring, T. Shepherd, and D. Waugh, Eds., SPARC Rep. 5, WCRP-30/2010, WMO/ TD-No. 40, 426 pp., https://www.sparc-climate.org/publications/ sparc-reports/sparc-report-no-5/.

Sun, L., W. A. Robinson, and G. Chen, 2012: The predictability of stratospheric warming events: More from the troposphere or the stratosphere? J. Atmos. Sci., 69, 768-783, https://doi.org/ 10.1175/JAS-D-11-0144.1.

Taguchi, M., T. Yamaga, and S. Yoden, 2001: Internal variability of the troposphere-stratosphere coupled system simulated in a simple global circulation model. J. Atmos. Sci., 58, 3184-3203, https://doi.org/10.1175/1520-0469(2001)058<3184: IVOTTS $>2.0 . \mathrm{CO} ; 2$.

Whitaker, J. S., and R. M. Dole, 1995: Organization of storm tracks in zonally varying flows. J. Atmos. Sci., 52, 1178-1191, https://doi.org/10.1175/1520-0469(1995)052<1178:OOSTIZ $>2.0 . \mathrm{CO} ; 2$.

Wu, Z., and T. Reichler, 2018: Towards a more Earth-like circulation in idealized models. J. Adv. Model. Earth Syst., 10, 1458-1469, https://doi.org/10.1029/2018MS001356. 